\title{
Single cell Raman spectroscopy to identify different stages of proliferating human hepatocytes for cell therapy
}

\author{
Chen Ma ${ }^{1,2}$, Ludi Zhang ${ }^{3,4}$, Ting He ${ }^{5}$, Huiying Cao ${ }^{1,2}$, Xiongzhao Ren ${ }^{6}$, Chenhui Ma ${ }^{1,2}$, Jiale Yang ${ }^{1,2}$, \\ Ruimin Huang ${ }^{1,2^{*}}$ (D) and Guoyu Pan ${ }^{1,2^{*}}$ (D)
}

\begin{abstract}
Background: Cell therapy provides hope for treatment of advanced liver failure. Proliferating human hepatocytes (ProliHHs) were derived from primary human hepatocytes $(\mathrm{PHH})$ and as potential alternative for cell therapy in liver diseases. Due to the continuous decline of mature hepatic genes and increase of progenitor like genes during ProliHHs expanding, it is challenge to monitor the critical changes of the whole process. Raman microspectroscopy is a noninvasive, label free analytical technique with high sensitivity capacity. In this study, we evaluated the potential and feasibility to identify ProliHHs from PHH with Raman spectroscopy.

Methods: Raman spectra were collected at least 600 single spectrum for PHH and ProliHHs at different stages (Passage 1 to Passage 4). Linear discriminant analysis and a two-layer machine learning model were used to analyze the Raman spectroscopy data. Significant differences in Raman bands were validated by the associated conventional kits.

Results: Linear discriminant analysis successfully classified ProliHHs at different stages and PHH. A two-layer machine learning model was established and the overall accuracy was at $84.6 \%$. Significant differences in Raman bands have been found within different ProliHHs cell groups, especially changes at $1003 \mathrm{~cm}^{-1}, 1206 \mathrm{~cm}^{-1}$ and $1440 \mathrm{~cm}^{-1}$. These changes were linked with reactive oxygen species, hydroxyproline and triglyceride levels in ProliHHs, and the hypothesis were consistent with the corresponding assay results.
\end{abstract}

Conclusions: In brief, Raman spectroscopy was successfully employed to identify different stages of ProliHHs during dedifferentiation process. The approach can simultaneously trace multiple changes of cellular components from somatic cells to progenitor cells.

Keywords: Raman spectroscopy, Cell therapy, Identification, Proliferating human hepatocytes, Dedifferentiation

\section{Background}

Liver disease is widely concerned among global researchers with 2 million deaths every year [1]. A lot of liver diseases may lead to liver cirrhosis and cancer, which may trigger liver failure [2]. Orthotopic liver transplantation is currently the final solution for liver failure. Nevertheless,

\footnotetext{
*Correspondence: rmhuang@simm.ac.cn; gypan@simm.ac.cn

${ }^{1}$ Shanghai Institute of Materia Medica, Chinese Academy of Sciences, Shanghai 201203, China
}

Full list of author information is available at the end of the article the healthy liver doners are very limited. The emergence of cell therapy presents new opportunities for multiple liver diseases. It was reported that hepatocytes transplantation could relieve or even cure liver diseases [3-5]. Because primary human hepatocytes $(\mathrm{PHH})$ are unable to proliferate in vitro, the availability of qualified $\mathrm{PHH}$ in clinic become the bottleneck of hepatocytes transplantation.

During the past decades, multiple cell sources have been investigated for liver transplantation as alternative original author(s) and the source, provide a link to the Creative Commons licence, and indicate if changes were made. The images or other third party material in this article are included in the article's Creative Commons licence, unless indicated otherwise in a credit line to the material. If material is not included in the article's Creative Commons licence and your intended use is not permitted by statutory regulation or exceeds the permitted use, you will need to obtain permission directly from the copyright holder. To view a copy of this licence, visit http://creativecommons.org/licenses/by/4.0/. The Creative Commons Public Domain Dedication waiver (http://creativeco mmons.org/publicdomain/zero/1.0/) applies to the data made available in this article, unless otherwise stated in a credit line to the data. 
of PHH. For example, fetal liver progenitors, adult human liver stem cells, hematopoietic stem cells, mesenchymal stem cells, human pluripotent stem cells, and induced pluripotent stem cells [6-13]. It was reported that rat fetal liver progenitor cells could differentiate into hepatocytes and reduce fibrotic activity after transplantation. Its efficacy is better than rat primary hepatocytes [14]. However, it is unfeasible to obtain enough human liver progenitor cells in clinic, not to mention conduct any quality control.

Zhang et al. found Wnt3a appeared to be essential to the initiation of the proliferation of $\mathrm{PHH}$. The significant upregulation of Wnt target genes lead to the proliferation of $\mathrm{PHH}$ in vitro, and the proliferating human hepatocytes (ProliHHs) showed both hepatocyte and progenitor features and could be reverted to mature phenotypes and rescue liver failure mice with high efficiency [15]. ProliHHs mature hepatic markers will gradually decrease while progenitor associated markers will increase during the passage. The progenitorlike cells could be restored to mature status (matured ProliHHs) in 3D culture process. Qiao et al. indicated that the matured ProliHHs had comparable metabolic capacities and biliary excretion capacity [16]. Nevertheless, maturation of ProliHHs may not be necessary for cell therapy in liver diseases, liver progenitor cells could be a better option for cell transplantation.

Because the hepatocytes proliferating and dedifferentiation to ProliHHs is a continuous process, a quick and non-invasive quality control method is critical to ensure the quality of harvested ProliHHs for liver transplantation. Conventional techniques, such as RT-qPCR and Western blotting need a lot of cells and are time-consuming. Theoretically, flow cytometric could precisely measure the quantities of antigens in each cell, however, they must be labelled with specific antibodies and/or fluorescently labelled microbeads [17].

Raman microspectroscopy has unique advantages in the analysis of multiple biological substances [18, 19]. The inelastic scattered light is collected to reflect biochemical molecules. It is nondestructive, noninvasive, label free, high sensitivity and resolution [20-22]. A single spectrum could be obtained in a few seconds which include information about multiple substances such as nucleic acids, proteins, lipids, saccharides and so on. In addition, the subcellular structures can be visualized by Raman image as well. For example, the accumulation of lipid droplets was observed in mice hepatocytes during nonalcoholic fatty liver disease (NAFLD) development [23]. Furthermore, Raman spectroscopy allows to monitor the various components at the single cell level which is suitable to provide cell heterogeneous information [24]. The analytical technique has been used to monitor cell metabolism, cell sorting, disease diagnosis and other related fields [25-31].

In this study, an established in-house protocol was employed to help $\mathrm{PHH}$ dedifferentiated into ProliHHs. $\mathrm{PHH}$ is defined as ProliHHs passage 0. ProliHHs at different stages (from passage 0 to passage 4, the later generation is closer to progenitor-like cells) were utilized to collect Raman spectra. The supervised linear discriminant analysis (LDA) was able to reduce the high dimensionality of data and distinguish three clearly clusters. Furthermore, the significant difference bands were semiquantified and attributed to the corresponding biomolecules. The Raman bands related to phenylalanine, hydroxyproline and lipids were chosen as representative biomarkers to verify the reliability of Raman. reactive oxygen species (ROS), hydroxyproline and triglyceride levels were measured from $\mathrm{PHH}$ to ProliHHs (P1 and P4). The results of the two methods are consistent. Finally, the machine learning model was successfully established and used to rapidly identify cells stages with an overall accuracy of $84.6 \%$. Importantly, it is the first time to indication that the changes of biochemical components during the dedifferentiation process from somatic cells (primary human hepatocytes) to progenitor like cells (ProliHHs) by Raman spectroscopy.

\section{Methods \\ Cell culture}

The primary human hepatocytes (Lot: 005 and Lot: 201678901, Novabiosis) were used to induced ProliHHs in the study. Cryopreserved PHH were thawed and seeded into Matrigel (Corning)-coated 6-well culture plates at 200,000 viable cells per well. After 6 h, cell medium was replaced by $\mathrm{HM}$ as previously published protocols. HM was mixed by $500 \mathrm{ml}$ Advanced DMEM/F-12 (Life Technologies), $1 \times \mathrm{N} 2$ supplement 100 (Life Technologies), $1 \times$ B27 Supplement 50 minus vitamin A (Life Technologies), $1 \mathrm{mM}$ N-acetyl-cysteine (Sigma-Aldrich), $10 \mathrm{mM}$ Nicotinamide (Solarbio), $2 \mathrm{ng} / \mathrm{ml}$ Recombinant humanFGF10 (Peprotech), 50 ng/ml Recombinant human EGF (Peprotech), $25 \mathrm{ng} / \mathrm{ml}$ Recombinant human HGF (Peprotech), $10 \mathrm{nM}$ Human [Leu15]-gastrin I (Sigma-Aldrich), 5uM A 83-01 (Tocris Bioscience), 10uM Rho kinase inhibitor Y-27632 (Selleck), $50 \mathrm{ng} / \mathrm{ml} \mathrm{Wnt3a} \mathrm{protein} \mathrm{(stemim-}$ mune LLC), 1\% Fetal bovine serum (Ausbian) [15]. The cell culture medium was changed every 3 days. After 7 days, $\mathrm{PHH}$ were successfully induced ProliHHs by HM and $2 \%$ hypoxic culture. Cells were washed with PBS and trypsinized for passaging when they reached $90 \%$ confluence. ProliHHs were incubated in $37^{\circ} \mathrm{C}$, hypoxia $\left(5 \% \mathrm{CO}_{2}\right.$, $2 \% \mathrm{O}_{2}$ ) incubator. ProliHHs (P0, P1 and P4) morphology were performed by phase contrast microscopy after cultured 1 day. HepG2 cells were obtained from ATCC. 
293FT cells were provided by Professor Lijian, Hui (State Key Laboratory of Cell Biology, CAS Center for Excellence in Molecular Cell Science, Shanghai Institute of Biochemistry and Cell Biology, Chinese Academy of Sciences, University of Chinese Academy of Science). Hepatoblast cells were provided by Professor Xin Cheng (State Key Laboratory of Cell Biology, CAS Center for Excellence in Molecular Cell Science, Institute of Biochemistry and Cell Biology, University of Chinese Academy of Sciences, Chinese Academy of Sciences). Primary rat hepatocytes were isolated from SD rats according to previous protocol [32].

\section{RT-qPCR analysis}

ProliHHs (P0, P1 and P4) were maintained on Matrigelcoated 12-well plates at 250,000 viable cells per well. After $24 \mathrm{~h}$, samples were collected by TRIzol reagent (Life Technology) and the EZ-10 Spin column \& Collection Tubes (Sangon Biotech). 500 ng RNA was reversely transcribed to cDNA using Hifair ${ }^{\circledR}$ III 1st Strand cDNA Synthesis SuperMix (Yeasen Biotech). cDNA was amplified by Hieff ${ }^{\circledR}$ qPCR SYBR Green Master Mix (Yeasen Biotech) on the Applied Biosystems 7500 Fast real-time PCR System (Thermo Fisher Scientific). Primers sequences were listed in Table S1. The relative mRNA levels were normalized by GAPDH. Each sample was performed in 3 replicates. GraphPad Prism 8.0 software was used to analyze data. The results represent means $\pm \mathrm{SD}$. One-way ANOVA was used for statistical analysis, ns $p \geq 0.05$, * $p<0.05, * * p<0.01,{ }^{* * * *} p<0.001, * * * * 0<0.0001$.

\section{Reactive oxygen species measurements}

ProliHHs (P0, P1 and P4) were seeded on Matrigelcoated 96-well plates at 50,000 viable cells per well and incubated overnight. Cells were washed in serum-free medium and treated with 10 mol $\mu / \mathrm{L}$ DCFH-DA (Beyotime Biotechnology) for $20 \mathrm{~min}$ at $37^{\circ} \mathrm{C}$. Then cells were washed 3 times in serum-free medium and measured fluorescence intensity at $488 \mathrm{~nm}$ excitation and $525 \mathrm{~nm}$ emission by the automatic microplate reader (Biotek).

\section{Triglyceride measurements}

ProliHHs (P0, P1 and P4) were cultured on Matrigelcoated 96-well plates at 50,000 viable cells per well. Cells were washed with PBS and removed supernatant. Then, $10 \mu$ l RIPA (Beyotime Biotechnology) was added into cells per well for $10 \mathrm{~min}$. Lysate was determined protein content by Take3 Micrometer plate (BioTek) and TG levels by Triglyceride assay kit (Nanjing Jiancheng Bioengineering Institute) according to manufacturer's instructions.

\section{Hydroxyproline measurements}

ProliHHs (P0, P1 and P4) were maintained on Matrigelcoated 12-well plates at 1000,000 viable cells per well. After $24 \mathrm{~h}$, the supernatant was collected and the hydroxyproline concentration was measured by Hydroxyproline assay kit (Nanjing Jiancheng Bioengineering Institute) according to manufacturer's instructions.

\section{Raman spectroscopy measurements}

ProliHHs (P0, P1 and P4) were seeded on 8-Well Chamber Raman Scattering Microslide (D-BAND) as cell density 100,000 cells $/ \mathrm{ml}$ for $24 \mathrm{~h}$. Cells were washed with PBS, fixed with $4 \%$ paraformaldehyde (Beyotime Biotechnology) for $15 \mathrm{~min}$, washed 3 times with sterile water. Then, the cells on Raman Scattering Microslide were air dried. Raman measurements were performed with a confocal Raman imaging system (alpha 300 R, WITec). The system was included in a $532 \mathrm{~nm}$ laser, 1800 grooves/ mm grating $(B L Z=500 \mathrm{~nm})$ and a CCD camera. Raman spectra (from 300 to $1800 \mathrm{~cm}^{-1}$ ) were collected by a 100 $\times$ objective $($ N.A. $=0.9)$ with laser power $9 \mathrm{~mW}$, integration time $10 \mathrm{~s}$ and accumulation number 1 . Calibration was performed using a silicon plate with its unique peak located at $520.7 \mathrm{~cm}^{-1}$. Raman spectra for each cell were randomly acquired within the cytoplasm $(n=5)$ and on the periphery $(n=5)$, respectively, based on the brightfield photo. A representative image with different focusing locations of laser was shown in Additional file 1: Figure S1. For each cell type, Raman spectra $(n=600$ in total) were acquired from 20 single cells per batch and 3 replicate batches. All spectra were normalized with subtraction of cosmic ray, baseline correction and area normalization.

\section{Raman data analysis}

All Raman data analysis were done under an R 3.6.3 environment with inhouse scripts. Linear discriminant analysis (LDA) and Principal component analysis (PCA) were used to reduce data dimensions and visualize classification. The Raman bands area were integrated to semiquantitative associated with biomolecules. The results represent median. The Student's t-test was used for statistical analysis, ns $p \geq 0.05, * p<0.05, * *<<0.01$, **** $p<0.001$, **** $p<0.0001$. Machine learning models were applied to build databases to rapidly identify cells. $75 \%$ data was used as a training set to establish model, $25 \%$ as a test set to evaluate model. It was used to build model by tenfold cross validation with five repetitions. Primarily, single classifiers were measured by k-nearest neighbor, linear discriminant analysis, partial leastsquares regression, linear support vector machine, radial basis function kernel support vector machine and 
random forest. In order to improve the accuracy of prediction, total of single classifiers were stacked together forming a two-layer model by GBM algorithm. The characteristics of the second layer is the single model results (KNN, LDA, PLS, Linear-SVM, RBF-SVM and RF), according to Hsu et al. protocol [33].

\section{Results}

\section{Generation and characterization of ProliHHs}

Previous researches showed that ProliHHs were biphenotypical cells which expressed genes of mature hepatocytes and liver progenitors [15]. To some extent, ProliHHs could replace primary human hepatocytes for drug safety evaluation and cell therapy [16]. However, there were a lot of gene expression and function differences among PHH and different passages of ProliHHs. Thus, a quick and simple approach to identify cells stages is urgently needed. Here, $\mathrm{PHH}$ were induced to ProliHHs by $\mathrm{HM}$ medium and hypoxia cultured for $7 \mathrm{~d}$. PHH could expand more than 250 folds to ProliHHs at P4. PHH and P1were polygonal, P4 partly were long strips. (Fig. 1a). SOX9, as a biomarker for liver progenitor cells, mRNA expression was gradually increased from $\mathrm{PHH}$ to ProliHHs (P1 and P4). The nuclear receptors FXR and CAR, mature hepatic genes, expressed in $\mathrm{PHH}, \mathrm{P} 1$ and $\mathrm{P} 4$. Interestingly, the FXR level of $\mathrm{P} 4$ was higher than $\mathrm{PHH}$. The efflux transporter MRP2 mRNA level was downregulated both P1 and P4 relative to PHH. Although ProliHHs expressions of Phase I drug metabolizing enzymes, including CYP3A4 and CYP2B6, were lower than PHH. The CYP2B6 gene of $\mathrm{P} 4$ showed nearly $1 / 3$ recovery compared to PHH. Phase II drug metabolizing enzymes, such as UGT1A1 and UGT2B7, were also expression of P1 and P4. In particular, the UGT1A1 level was significantly higher than PHH (Fig. 1b). In brief, from gene expression perspective, ProliHHs only maintained part of drug metabolic and transport genes, the cells expressed more and more liver progenitor biomarkers from P1 to P4.

\section{Raman spectroscopy and classification analysis}

Although the mRNA and protein levels could characterize the differences between $\mathrm{PHH}$ and different passages of ProliHHs, those methods cost much time while get limited information. Therefore, we evaluated the potential of collected Raman spectroscopy to monitor cell to cell heterogeneity. PHH, ProliHHs P1 and P4 were respectively examined at least 600 single spectrum (Table 1). The fingerprint region, including more than $90 \%$ of the cellular peaks, was collected to identify cell stages [34]. The averaged spectra of each type cell were showed in Fig. 2a. Principal component analysis (Additional file 1: Fig. S1) and linear discriminant analysis (Fig. 2b) were used to reduce dimension and highlight the spectral signatures on different cell stages. One Raman sample was represented as a point, at least 600 samples to reflect each type cells in different figures. Obviously, LDA was able to discriminate cell populations better than PCA. The significant wavenumbers in LD1 and LD2 contributed to differences among three groups were provided in (Additional file 1: Fig. S2). This suggests the ability of Raman spectroscopy to classify cells.

\section{Machine learning models}

In addition to extract significant wavenumbers, it is suitable to establish Raman dataset by machine learning models. The entire Raman spectral (1850) was randomly divided into two parts, $75 \%$ to establish the training set, $25 \%$ to verify the model. First, the single model KNN, LDA, PLS, Linear-SVM, RBF-SVM and RF were respectively constructed to classify by tenfold cross-validation with five repetitions and the corresponding parameters were showed in Additional file 2: Table S2. The overall accuracy was $83.8 \%$ in Linear-SVM model. Then, the fitted results of above six models were stacked to form a two-layer machine learning model, which improved the prediction accuracy to $84.6 \%$ (Table 2). The sensitivities were $75.6 \%, 88.7 \%, 89.7 \%$ and specificity were $90.2 \%$, 95.5\%, 81.2\% for PHH, P1 and P4.

\section{Potential biomarkers of cell identification}

There were numbers of differences in Raman bands which may reflect cell biochemical components changes from PHH to different stages of ProliHHs (P1 and P4) (Fig. 3). The results suggested significant differences among spectral bands of cell clusters located at $480 \mathrm{~cm}^{-1}$ (glycogen), $831 \mathrm{~cm}^{-1}$ (tyrosine), $840-860 \mathrm{~cm}^{-1}$ (polysaccharide), $1003 \mathrm{~cm}^{-1}$ (phenylalanine), $1080 \mathrm{~cm}^{-1}$ (amide II, typical phospholipids), $1172 \mathrm{~cm}^{-1}$ (C-H in plane bending mode of tyrosine), $1206 \mathrm{~cm}^{-1}$ (hydroxyproline),1265 $\mathrm{cm}^{-1}$ ( $\alpha$-helix, collagen, tryptophan), $1300 \mathrm{~cm}^{-1}$ (lipids), $1337 \mathrm{~cm}^{-1}$ (Amide III), $1440 \mathrm{~cm}^{-1}$ $\left(\mathrm{CH}_{2}\right.$ and $\mathrm{CH}_{3}$ formation vibrations of lipids), $1658 \mathrm{~cm}^{-1}$ (Amide I of proteins), $1744 \mathrm{~cm}^{-1}$ (carbonyl feature of lipid spectra) respectively. To further quantify the changes in 3 groups, relative Raman bands area were integrated (Fig. $4 \mathrm{a}-\mathrm{m})$. The peak at $480 \mathrm{~cm}^{-1}(p \leq 0.001)$ and $831 \mathrm{~cm}^{-1}(p \leq 0.001)$ were significant difference between $\mathrm{PHH}$ and ProliHHs (P1 and P4). The Raman bands area at $840-860 \mathrm{~cm}^{-1}(p \leq 0.0001), 1080 \mathrm{~cm}^{-1}(p \leq 0.0001)$, $1265 \mathrm{~cm}^{-1}(p \leq 0.001), 1300 \mathrm{~cm}^{-1}(p \leq 0.0001), 1440 \mathrm{~cm}^{-1}$ $(p \leq 0.0001), 1658 \mathrm{~cm}^{-1}(p \leq 0.0001)$ and $1744 \mathrm{~cm}^{-1}(p \leq$ $0.0001)$ were significantly decreased following ProliHHs derived and passaged. In contrast, the Raman bands area at $1003 \mathrm{~cm}^{-1}(p \leq 0.0001), 1206 \mathrm{~cm}^{-1}(p \leq 0.0001)$, $1337 \mathrm{~cm}^{-1}(p \leq 0.0001)$ were significantly increased. 


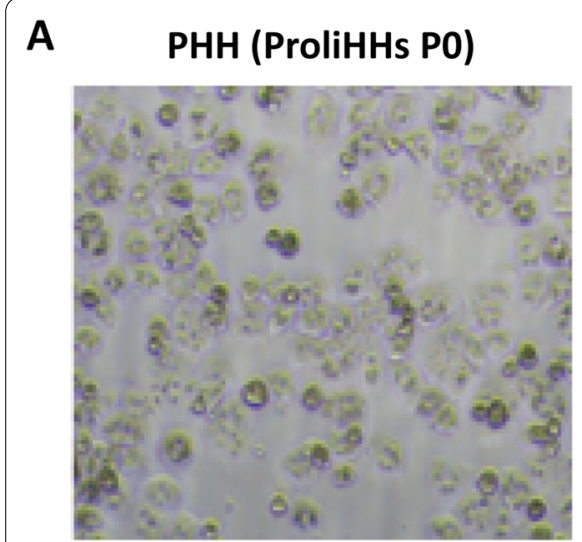

ProliHHs P1

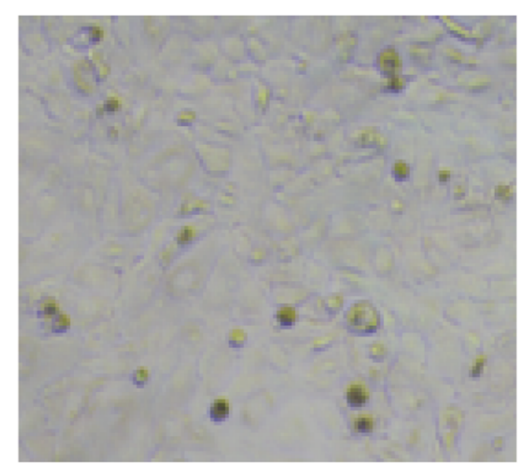

\section{ProliHHs P4}

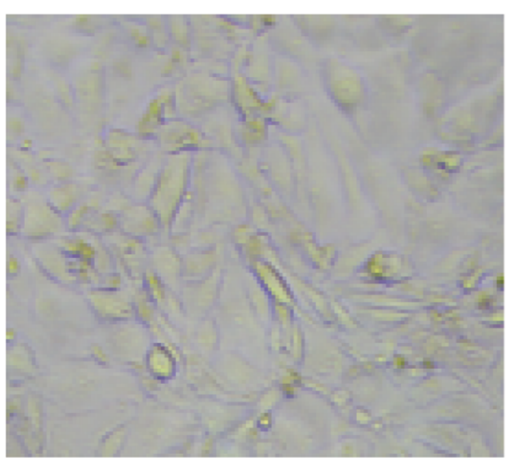

B

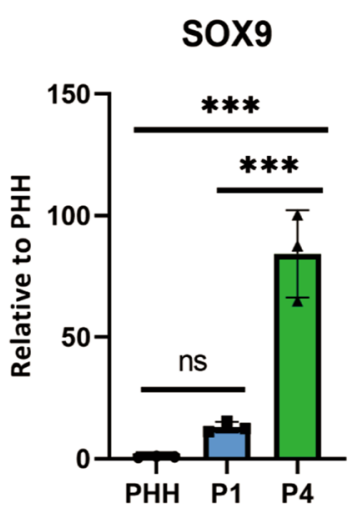

CYP3A4

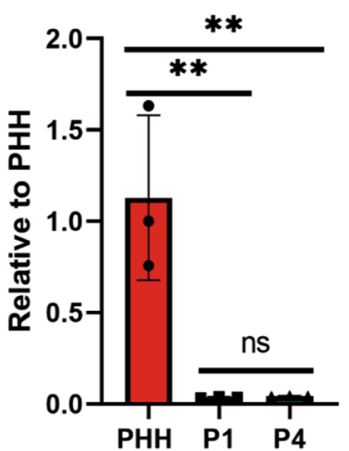

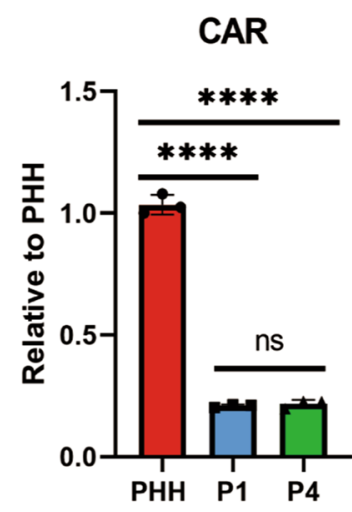

CYP2B6

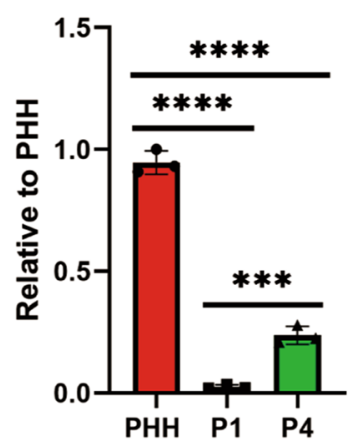

FXR

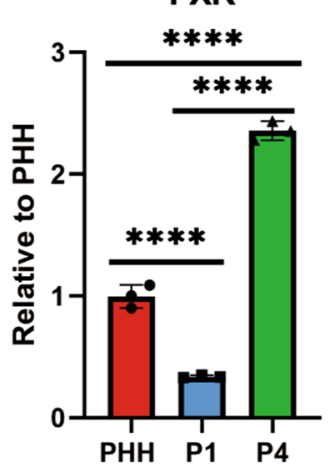

UGT1A1

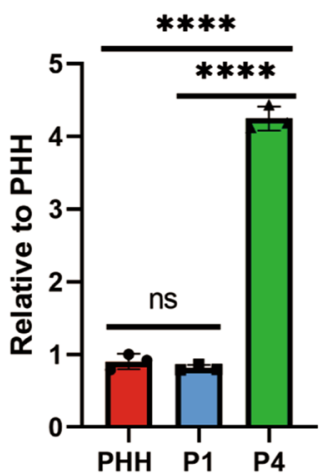

MRP2

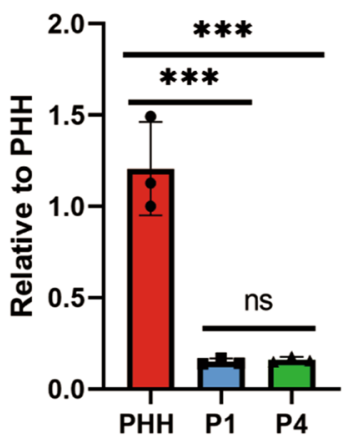

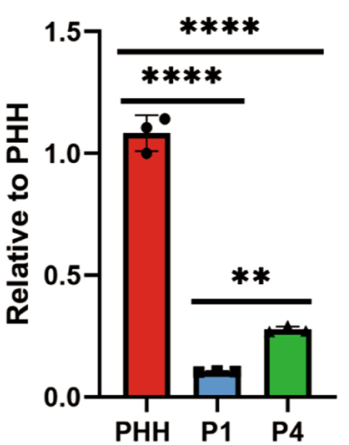

Fig. 1 ProliHHs were derived from PHH (Lot:005) and identified by mRNA. a Microscopic imaging of PHH, ProliHHs P1 and P4 (magnification, $\times$ 10). b The mRNA expressions of biomarkers relative to hepatocytes and progenitor cells in PHH, ProliHHs P1 and P4. The results represent means \pm SD, $\mathrm{ns} p \geq 0.05,{ }^{*} p<0.05,{ }^{* *} p<0.01,{ }^{* * *} p<0.001,{ }^{* * * *} p<0.0001$. (PHH: primary human hepatocytes, ProliHHs: proliferating human hepatocytes, P1: passage 1, P4: passage 4)

Although the band at $1172 \mathrm{~cm}^{-1}$ was no change between $\mathrm{PHH}$ and P1, P4 was higher than the others.

The Raman bands changes at $1003 \mathrm{~cm}^{-1}, 1206 \mathrm{~cm}^{-1}$ and $1440 \mathrm{~cm}^{-1}$ were identified with reactive oxygen species, hydroxyproline and triglyceride levels by the corresponding kit. The ROS levels significant down regulated from $\mathrm{PHH}$ to ProliHHs (P1 and P4), while there was no difference between P1 and P4 (Fig. 5a). TG concentration gradually decreased from PHH to ProliHHs (Fig. 5b). Hydroxyproline concentration increased during the dedifferentiation process, however there was no statistical difference (Fig. 5c). These findings indicated the potential biomarkers for cell quality control which is essential in cell therapy. 
Table 1 Numbers of Raman spectra acquired from $\mathrm{PHH}$, ProliHHs P1 and P4

\begin{tabular}{ll}
\hline Group & $\begin{array}{l}\text { Number } \\
\text { of } \\
\text { spectra }\end{array}$ \\
\hline PHH & 620 \\
ProliHHs P1 & 624 \\
ProliHHs P4 & 606 \\
\hline
\end{tabular}

\section{Discussion}

Previous researches have shown that PHH could gradually dedifferentiated from hepatocyte like to progenitor like state during the ProliHHs proliferating process $[15$, 16]. A non-invasive and comprehensive quality control method is obligated to link ProliHHs functions with the continuous process, which is critical to apply cell transplantation in clinic $[35,36]$. Raman microspectroscopy has superiority to obtain multi- information in a labelfree and rapid analysis for different kind of alive cells.

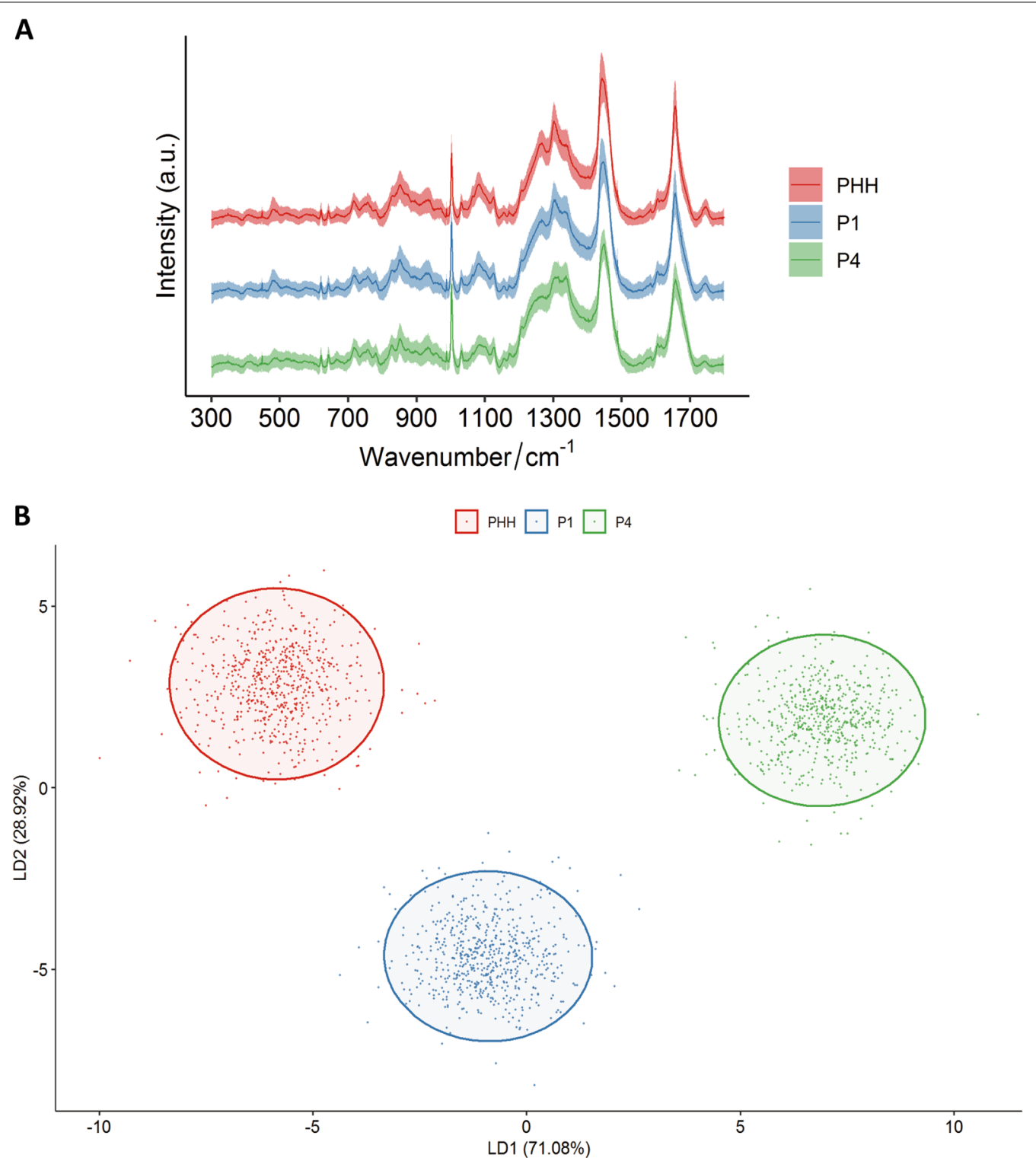

Fig. 2 Raman spectroscopy and classification analysis for PHH (Lot:005), ProliHHs P1 and P4. a The averaged spectra ( $n=1850$ ) collected by PHH ( $n=620)$, P1 ( $n=624)$ and P4 ( $n=606)$ on fingerprint region. b Linear discriminant analysis clearly distinguished three cell groups. (The red, blue, and green colors represent PHH, ProliHHs P1 and P4 cells, respectively. PHH: primary human hepatocytes, ProliHHs: proliferating human hepatocytes, P1: passage 1, P4: passage 4) 
Table 2 Machine learning by stacked (KNN, LDA, PLS, LinearSVM, RBF-SVM, RF) model to identify cells. Overall accuracy at $84.6 \%$

\begin{tabular}{llll}
\hline & Reference & & \\
\cline { 2 - 4 } & P1 & P4 & PHH \\
\hline $\begin{array}{lll}\text { Model prediction } \\
\text { ProliHHs P1 }\end{array}$ & 118 & 14 & 16 \\
ProliHHs P4 & 14 & 134 & 0 \\
PHH & 24 & 3 & 139 \\
Sensitivity (\%) & 75.6 & 88.7 & 89.7 \\
Specificity (\%) & 90.2 & 95.5 & 81.2 \\
\hline
\end{tabular}

Hence, we investigated the feasibility of Raman spectroscopy to capture the cell status in the production of the hepatocytes-derived progenitor like cells at single cell level.

ProliHHs were derived from $\mathrm{PHH}$ and passaged four times. The mRNA levels suggested that the mature hepatic function gradually down regulated and progenitor like function up regulated, which was consistent with previous reports $[15,16]$. At least 600 Raman spectral were collected for each passage stage. LDA clearly distinguished PHH, ProliHHs P1 and P4. P9 of ProliHHs were also collected and conducted similar analysis (Additional file 1: Fig. S3). It was found that the Raman spectra of ProliHHs P4 and P9 were close but distinguishable, which was consistent with the fact that ProliHHs was more like progenitor cells after P4 [15]. Therefore, P0 (PHH) to $\mathrm{P} 4$ of ProliHHs were used as a representative for the following research.

All Raman spectra were sufficiently used to set up a database by machine learning model. A two-layer model was successfully stacked by KNN, LDA, PLS, SVM-Linear, SVM-RBF and RF, the overall accuracy was $84.6 \%$. The results suggested it was feasible to identify ProliHHs status by comparing their Raman bands with database in a few seconds. It was reported that the Raman spectra collected from cell nuclei had the best signal to noise ratio [37]. Therefore, the samples collected

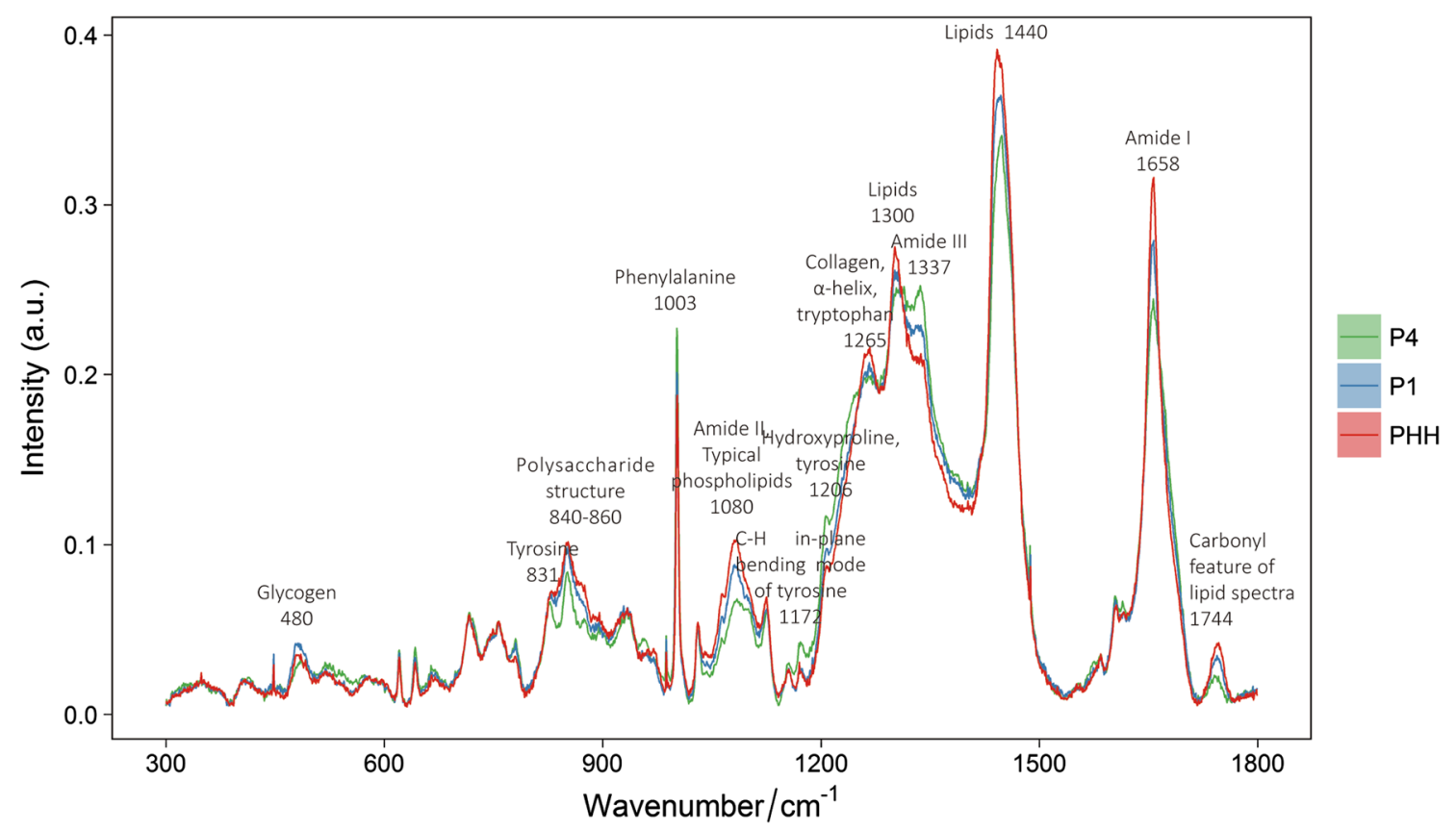

Fig. 3 The biochemical molecules represented by the specific Raman bands in the average spectral (Lot:005)

Fig. 4 The peak area were semi-quantitative to compare differences of the specific Raman bands $\mathbf{a} 480 \mathrm{~cm}^{-1}$ (glycogen), b $831 \mathrm{~cm}^{-1}$ (tyrosine), c $840-860 \mathrm{~cm}^{-1}$ (polysaccharide structure), d $1003 \mathrm{~cm}^{-1}$ (phenylalanine), e $1080 \mathrm{~cm}^{-1}$ (amide II, typical phospholipid), f $1172 \mathrm{~cm}^{-1}(\mathrm{C}-\mathrm{H}$ in-plane bending mode of tyrosine), $\mathbf{g} 1206 \mathrm{~cm}^{-1}$ (hydroxyproline, tyrosine), $\mathbf{h} 1265 \mathrm{~cm}^{-1}$ (a-helix, collagen, tryptophan), $\mathbf{i} 1300 \mathrm{~cm}^{-1}$ (lipids), $\mathbf{j} 1337 \mathrm{~cm}-1$ (amide III), $\mathbf{k} 1440 \mathrm{~cm}^{-1}$ (lipids), I $1658 \mathrm{~cm}^{-1}$ (amide I), $\mathbf{m} 1744 \mathrm{~cm}^{-1}$ (carbonyl feature of lipid spectra) in PHH (Lot:005), ProliHHs P1 and P4. The results represent median, $\mathrm{ns} p \geq 0.05,{ }^{*} p<0.05,{ }^{* *} p<0.01,{ }^{* * *} p<0.001,{ }^{* * *} p<0.0001$ (PHH: primary human hepatocytes, ProliHHs: proliferating human hepatocytes, P1: passage 1, P4: passage 4) 

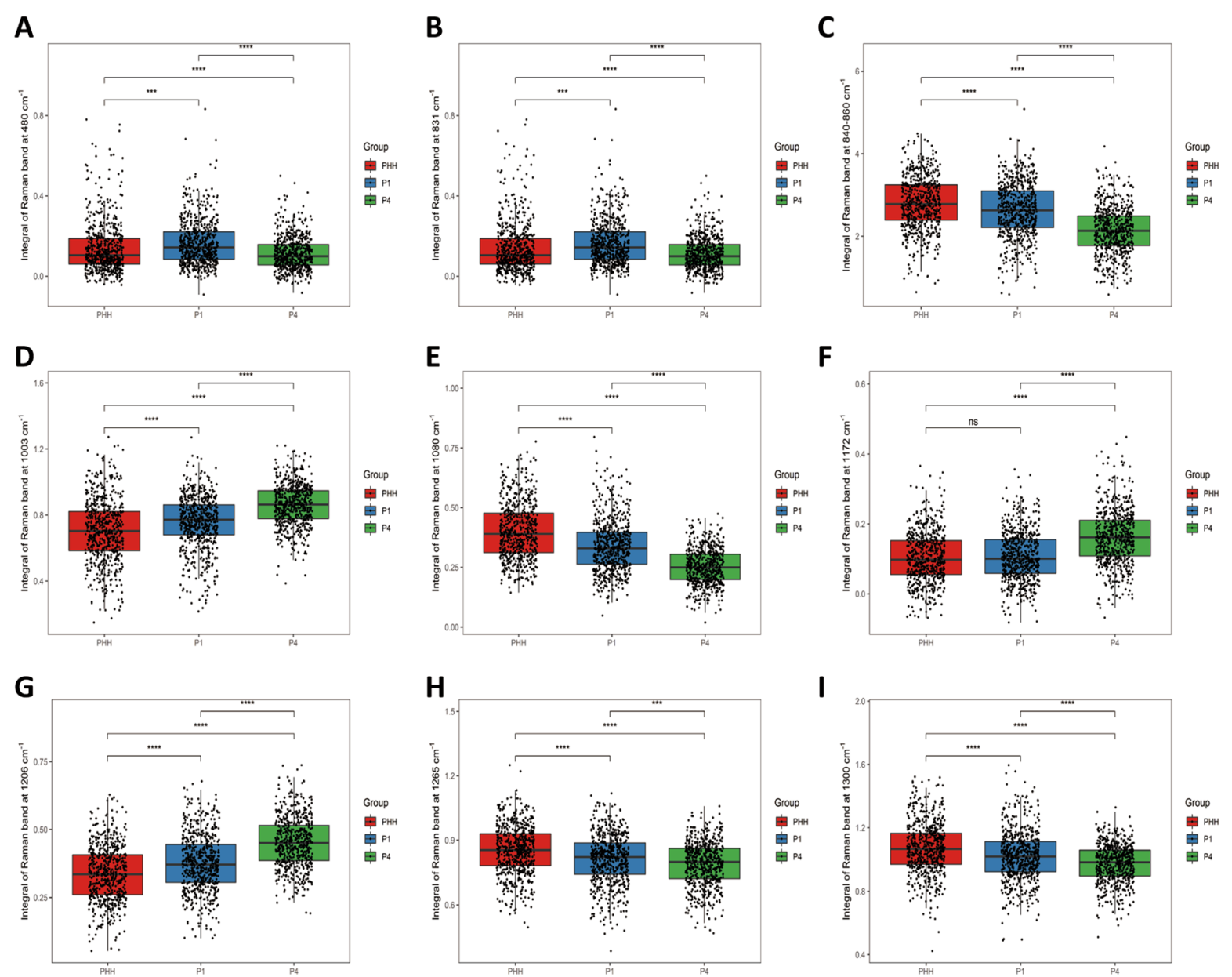

Group
PHH
P1
P4
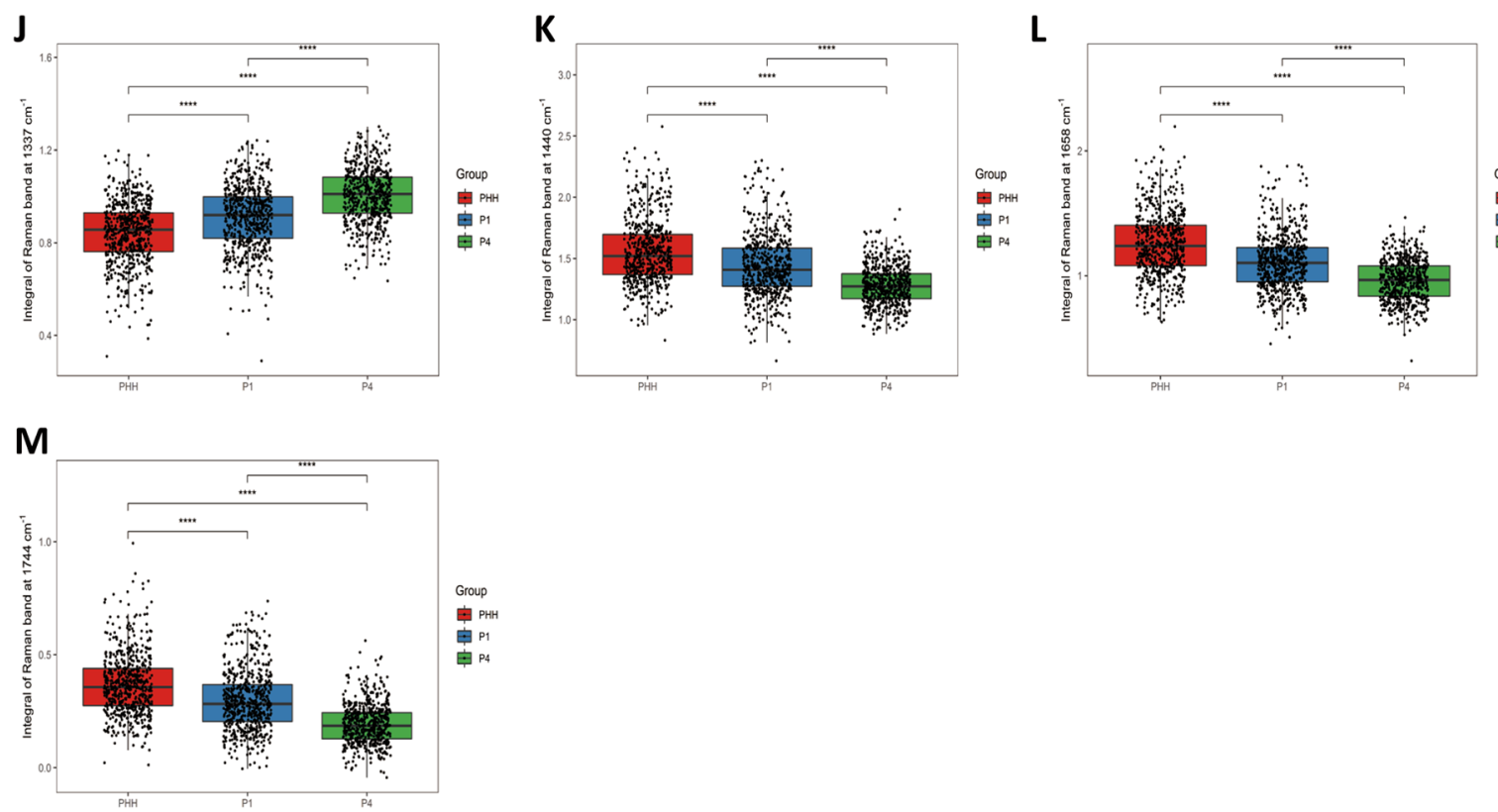

Fig. 4 (See legend on previous page.) 

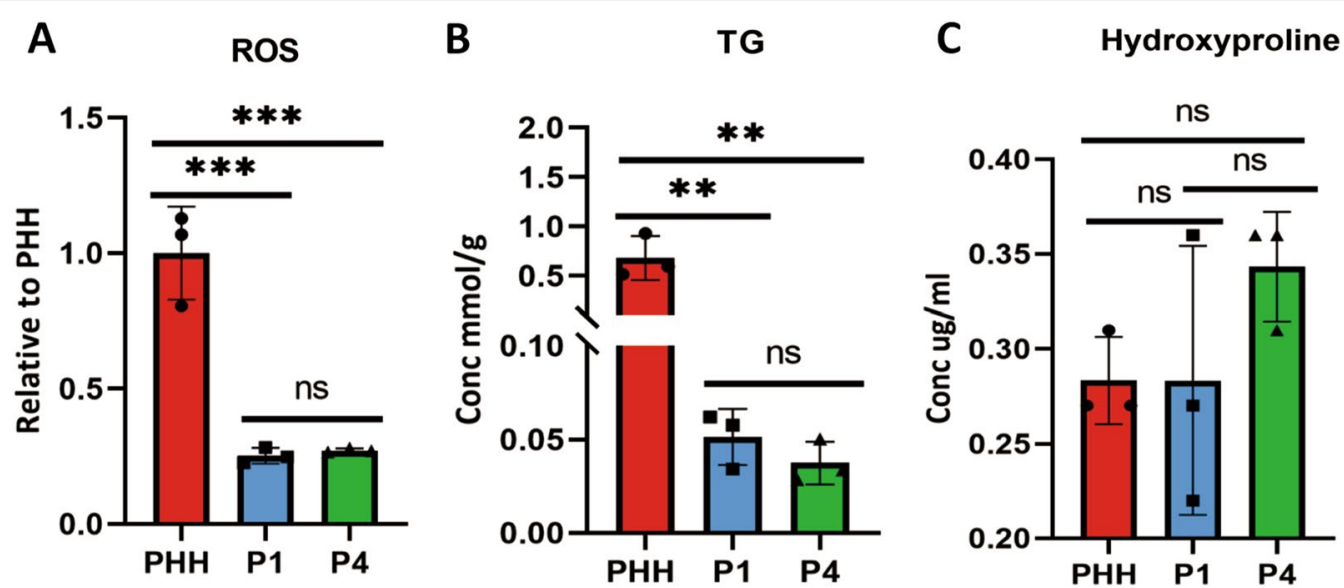

Fig. 5 The potential biomarkers to identify PHH (Lot:005), P1 and P4. a Reactive oxygen species (ROS) levels decreased $\mathbf{b}$ triglyceride (TG) concentration decreased and $\mathbf{c}$ hydroxyproline concentration increased from PHH to ProliHHs (P1 and P4). The results represent means \pm SD, ns $p \geq$ $0.05,{ }^{*} p<0.05,{ }^{* *} p<0.01,{ }^{* * *} p<0.001,{ }^{* * *} p<0.0001(\mathrm{PHH}$ : primary human hepatocytes, ProliHHs: proliferating human hepatocytes, P1: passage 1, P4: passage 4)

in cellular center and peripheral were separately built with machine learning models. The results showed that the overall accuracy was similar (Additional file 2: Table S3). It confirmed the robustness of our established Raman machine learning model, and the location of collection would not affect the prediction results. There is always a balance between robustness and accuracy. When accuracy increases, robustness decreases accordingly [38]. Our model achieved better robustness so that it can tolerate more variability between cell batches. The whole study was conducted twice by ProliHHs from similar donor PHH (Lot: 005, Novabiosis). Another similar study was conducted with different donor PHH (Lot: 201678901, Novabiosis). In all the three independent studies, $\mathrm{PHH}$ were able to induce ProliHHs successfully, and the corresponding Raman spectra were collected and analyzed. The machine learning models were able to identify different stages cells with high accuracy. The result was consistent and attached at Additional files 3 and 4, which confirmed the robustness of the model. And other types cells such as 293FT (human embryonic kidneys), HepG2 (human liver hepatocellular carcinoma) and PRH (primary rat hepatocytes) were collected Raman spectroscopy (Additional file 3: Figure S8). The average Raman spectra were significantly different among these cells.

Hepatocytes have various functions such as protein synthesis, biotransformation, energy storage and detoxification. However, its cellular components may change with the cell plasticity during cell dedifferentiation from $\mathrm{PHH}$ to ProliHHs [39]. It is not clear if Raman spectra could capture these changes. In this study, a lot of significant changes have been identified within Raman bands.
For example, we have discovered that the mitochondrial associated Raman band at $1003 \mathrm{~cm}^{-1}$ (phenylalanine) increased during $\mathrm{PH}$ dedifferentiation. The result suggested that the mitochondria decreased during cell dedifferentiation. In order to verify this, reactive oxygen levels were measured and it was found ROS levels in ProliHHs (P1 and P4) were much lower than PHH (Fig. 5a), which implied the mitochondrial activity was down regulated after $\mathrm{PHH}$ was converted to ProliHHs, progenitorlike cells. Actually, it was reported that in order to obtain proliferation, pluripotency and plasticity functions as progenitor-like cells, the reprogramming somatic cells became immature and the amount of mitochondrial DNA was reduced [40-42], which was consistent with our results.

The significant decrease of the Raman band related to lipids were identified at $1080 \mathrm{~cm}^{-1}, 1300 \mathrm{~cm}^{-1}$, $1440 \mathrm{~cm}^{-1}, 1744 \mathrm{~cm}^{-1}$ respectively. In order to verify the reliability of aforementioned results, triglycerides (TG) concentrations were determined, which was primary lipids storage form in hepatocyte like cells. The result revealed that the TG levels in ProliHHs (P1 and P4) were much lower than PHH (Fig. 5b), which was consistent with Raman measurement. Fu et al. also described that the lipids genes were down regulated when $\mathrm{PHH}$ was reprogrammed to liver progenitor like cells [43]. Therefore, the Raman lipids bands may be used as potential hallmarks of hepatocyte-like cell status, or even could be utilized to diagnose non-alcoholic fatty liver disease [44, 45].

Another interesting finding is, with the progress of dedifferentiation to later passages of ProliHHs, the hydroxyproline content significantly increased in Raman 
spectrum. Our current data revealed the increased hydroxyproline trend in $\mathrm{P} 4$ of ProliHHs (Fig. 5c). The up-regulated hydroxyproline may imply that the later passaged ProliHHs activated epithelial-mesenchymal transition (EMT) related pathways. Upon liver injury, mature hepatocytes can be reprogrammed to liver progenitor-like cells (LPLCs) in vivo. Notably, these LPLCs show significant induction of mesenchymal markers as well as progenitor markers, suggesting the activation of EMT pathway during the conversion $[39,46]$. A recent single-cell transcriptomics analysis also reveals the emergence of liver progenitors with mesenchymal features during liver development [47]. In line with these in vivo findings, it has been demonstrated that ProliHHs at late passages showed a tendency to express progenitorlike profiles and mesenchymal markers [15]. Therefore, the increased hydroxyproline can possibly be applied as a marker for the conversion of ProliHH to liver progenitors. However, this hypothesis required further verification.

\section{Conclusion}

In short, Raman spectra provided us a lot of information about the changes of biochemical molecules in the process of somatic cells dedifferentiation. To our knowledge, this work is the first report that Raman spectroscopy could successfully identify ProliHHs from P0 to P4. A machine learning model was established at overall accuracy of $84.6 \%$, which made it feasible to conduct real-time ProliHHs quality control for cell transplantation. Raman spectra could capture the change of ROS, hydroxyproline and lipids in hepatocyte-like cells, their changes were consistent with their physiological functions at different cellular status, respectively. Therefore, it is feasible to apply Raman spectra for the identification of progenitorlike cells from somatic cells and obtain multiple cellular components information simultaneously [48].

\begin{abstract}
Abbreviations
ProliHHs: Proliferating human hepatocytes; PHH: Primary human hepatocytes; LDA: Linear discriminant analysis; ROS: Reactive oxygen species; TG: Triglyceride; NAFLD: Nonalcoholic fatty liver disease; PCA: Principal component analysis; KNN: K-nearest neighbor; PLS: Partial least-squares regression; Linear-SVM: Linear support vector machine; RBF-SVM: Radial basis function kernel support vector machine; RF: Random forest; EMT: Epithelial-mesenchymal transition; LPLCs: Liver progenitor-like cells.
\end{abstract}

\section{Supplementary Information}

The online version contains supplementary material available at https://doi. org/10.1186/s13287-021-02619-9.

Additional file 1: Figure S1. The location of laser focusing $(+)$ at the center (A) and periphery (B) in ProliHHs P1 (Lot:005). Figure S2. Principal component analysis of all Raman spectra in PHH (Lot:005), ProliHHs P1 and P4 cells. (The red, blue, and green colors represent PHH, ProliHHs P1 and $\mathrm{P} 4$ cells, respectively. $\mathrm{PHH}$ : primary human hepatocytes, ProliHHs: proliferating human hepatocytes, P1: passage 1, P4: passage 4). Figure S3. $10 \%$ most significant wavenumbers in LD1 (A) and LD2 (B) contributing to differences among PHH (Lot:005), ProliHHs P1 and P4 cells. (PHH: primary human hepatocytes, ProliHHs: proliferating human hepatocytes, P1: passage 1, P4: passage 4, LD: Linear discriminant). Figure S4. LDA analysis of all Raman spectra in PHH (Lot:005), P1, P4 and P9 cells. (LDA: Linear discriminant analysis, $\mathrm{PHH}$ : primary human hepatocytes, ProliHHs: proliferating human hepatocytes, P1: passage 1, P4: passage 4, P9: passage 9)

Additional file 2: Table S1. Primers for QPCR. Table S2. Machine learning model (A) KNN, (B) LDA, (C) PLS, (D) Linear-SVM, (E) RBF-SVM, (F) Random forest to identify cells. Overall accuracy at $62.6 \%, 67.5 \%, 63.6 \%, 83.8 \%$, $81.4 \%, 65.6 \%$. Table S3. Machine learning by stacked (KNN, LDA, PLS, SVMLinear, SVM-RBF, RF) model to identify cells from center (A) and peripheral (B) Overall accuracy at $83.4 \%, 82.33 \%$.

Additional file 3: Figure S5. Raman spectroscopy and classification analysis for PHH (Lot:201678901), ProliHHs P1 and P4. (A) The averaged spectra $(n=1829)$ collected by PHH $(n=619), \mathrm{P} 1(n=595)$ and P4 $(n=615)$ on fingerprint region. (B) Linear discriminant analysis clearly distinguished three cell groups. (The red, blue, and green colors represent PHH, ProliHHs P1 and P4 cells, respectively. PHH: primary human hepatocytes, ProliHHs: proliferating human hepatocytes, P1: passage 1, P4: passage 4). Figure S6. The biochemical molecules represented by the specific Raman bands in the average spectral (Lot:201678901). Figure S7. The peak area were semi-quantitative to compare differences of the specific Raman bands (A) $480 \mathrm{~cm}^{-1}$ (glycogen), (B) $831 \mathrm{~cm}^{-1}$ (tyrosine), (C) $840-860 \mathrm{~cm}^{-1}$ (polysaccharide structure), (D) $1003 \mathrm{~cm}^{-1}$ (phenylalanine), (E) 1080 $\mathrm{cm}^{-1}$ (amide II, typical phospholipid), (F) $1172 \mathrm{~cm}^{-1}$ (C-H in-plane bending mode of tyrosine), (G) $1206 \mathrm{~cm}^{-1}$ (hydroxyproline, tyrosine), (H) $1265 \mathrm{~cm}^{-1}$ (a-helix, collagen, tryptophan), (I) $1300 \mathrm{~cm}^{-1}$ (lipids), (J) $1337 \mathrm{~cm}^{-1}$ (amide III), (K) $1440 \mathrm{~cm}^{-1}$ (lipids), (L) $1658 \mathrm{~cm}^{-1}$ (amide I), (M) $1744 \mathrm{~cm}^{-1}$ (carbonyl feature of lipid spectra) in PHH (Lot:201678901), ProliHHs P1 and P4. The results represent median, ns $p \geq 0.05,{ }^{*} p<0.05$, ${ }^{* *}$ $p<0.01$, ${ }^{* * *} p<0.001,{ }^{* * * *} p<0.0001$. (PHH: primary human hepatocytes, ProliHHs: proliferating human hepatocytes, P1: passage 1, P4: passage 4). Figure S8. The average Raman spectral of PHH (Lot: 201678901, $n=208$ ), PRH $(n=201)$, HepG2 $(n=204)$ and 293FT ( $n=205)$. (PHH: primary human hepatocytes, PRH: primary rat hepatocytes, HepG2: human liver hepatocellular carcinoma, 293FT: human embryonic kidneys.). Table S4 Machine learning by stacked (KNN, LDA, PLS, Linear-SVM, RBF-SVM, RF) model to identify cells. Overall accuracy at 81.32\% (Lot: 201678901).

Additional file 4: Figure S9. Raman spectroscopy and classification analysis for PHH (Lot:005), ProliHHs P1, P4 and hepatoblast. (A) The averaged spectra $(n=815)$ collected by PHH $(n=204)$, P1 $(n=202)$, P4 $(n=202)$ and hepatoblast $(n=207)$ on fingerprint region. (B) Linear discriminant analysis clearly distinguished three cell groups. (The red, blue, green and purple colors represent PHH, ProliHHs P1, P4 and hepatoblast cells, respectively. PHH: primary human hepatocytes, ProliHHs: proliferating human hepatocytes, P1: passage 1, P4: passage 4). Figure S10. The biochemical molecules represented by the specific Raman bands in the average spectral (Lot:005). Figure S11. The peak area were semi-quantitative to compare differences of the specific Raman bands (A) $480 \mathrm{~cm}^{-1}$ (glycogen), (B) $831 \mathrm{~cm}^{-1}$ (tyrosine), (C) $840-860 \mathrm{~cm}^{-1}$ (polysaccharide structure), (D) $1003 \mathrm{~cm}^{-1}$ (phenylalanine), (E) $1080 \mathrm{~cm}^{-1}$ (amide II, typical phospholipid), (F) $1172 \mathrm{~cm}^{-1}$ (C-H in-plane bending mode of tyrosine), (G) 1206 $\mathrm{cm}^{-1}$ (hydroxyproline, tyrosine), (H) $1265 \mathrm{~cm}^{-1}$ (a-helix, collagen, tryptophan), (I) $1300 \mathrm{~cm}^{-1}$ (lipids), (J) $1337 \mathrm{~cm}^{-1}$ (amide III), (K) 1440 $\mathrm{cm}^{-1}$ (lipids), (L) $1658 \mathrm{~cm}^{-1}$ (amide I), (M) $1744 \mathrm{~cm}^{-1}$ (carbonyl feature of lipid spectra) in PHH (Lot:005), ProliHHs P1, P4 and hepatoblast. The results represent median, ns $p \geq 0.05,{ }^{*} p<0.05,{ }^{* *} p<0.01$, ${ }^{* * *} p<0.001$, ${ }_{* * * *} p<0.0001$. (PHH: primary human hepatocytes, ProliHHs: proliferating human hepatocytes, P1: passage 1, P4: passage 4). Table S5 Machine learning by stacked (KNN, LDA, PLS, Linear-SVM, RBF-SVM, RF) model to identify cells. Overall accuracy at $92.08 \%$ (Lot: 005).

\section{Acknowledgements}

Not applicable. 


\section{Authors' contributions}

CM, GYP and RMH designed the research. CM, LDZ, TH, HYC, XZR, CHM and JLY performed the experiments. CM and LDZ analyzed and interpreted the data. CM and GYP were major contributors in writing the manuscript. All authors read and approved the final manuscript.

\section{Funding}

This study was supported by the 'Organ Reconstruction and Manufacturing' Strategic Priority Research Program of the Chinese Academy of Sciences (Grant number XDA16020205), the National Science Foundation of China (Grant numbers 81872927, 81771890, 91859106), the International Partnership Program of Chinese Academy of Sciences (Grant number 153631KYSB20160004), the Independent Deployment Program of the Institute of Pharmaceutical Innovation of the Chinese Academy of Sciences (Grant number CASIMM0120184005), the research fund from Shanghai Science and Technology Committee (Grant number 20S11901400), and Shanghai Municipal Science and Technology Major Project. We thank technical supports from the Institutional Center for Shared Technologies and Facilities of Shanghai Institute of Materia Medica, Chinese Academy of Sciences.

\section{Availability of data and materials}

The datasets used and/or analysed during the current study are available from the corresponding author on reasonable request.

\section{Declarations}

\section{Ethics approval and consent to participate}

Not applicable.

\section{Consent for publication}

Not applicable.

\section{Competing interests}

The authors declare that they have no competing interests.

\section{Author details}

1Shanghai Institute of Materia Medica, Chinese Academy of Sciences, Shanghai 201203, China. ${ }^{2}$ University of Chinese Academy of Sciences, Beijing 100049, China. ${ }^{3}$ State Key Laboratory of Cell Biology, CAS Center for Excellence in Molecular Cell Science, Shanghai Institute of Biochemistry and Cell Biology, Chinese Academy of Sciences, Shanghai, China. ${ }^{4}$ University of Chinese Academy of Science, Beijing, China. ${ }^{5}$ School of Pharmaceutical Sciences, Nanjing Tech University, Nanjing, China. 'State Key Laboratory of Cell Biology, CAS Center for Excellence in Molecular Cell Science, Institute of Biochemistry and Cell Biology, University of Chinese Academy of Sciences, Chinese Academy of Sciences, Shanghai 200031, China.

Received: 12 April 2021 Accepted: 23 July 2021

Published online: 30 October 2021

\section{References}

1. Asrani SK, et al. Burden of liver diseases in the world. J Hepatol. 2019;70(1):151-71.

2. Wang FS, et al. The global burden of liver disease: the major impact of China. Hepatology. 2014;60(6):2099-108.

3. Dwyer BJ, et al. Cell therapy for advanced liver diseases: repair or rebuild. J Hepatol. 2021;74(1):185-99.

4. Enosawa $\mathrm{S}$, et al. Hepatocyte transplantation using a living donor reduced graft in a baby with ornithine transcarbamylase deficiency: a novel source of hepatocytes. Liver Transpl. 2014;20(3):391-3.

5. Fisher RA, et al. Defining hepatocellular chimerism in a liver failure patient bridged with hepatocyte infusion. Transplantation. 2000;69(2):303-7

6. Messina, A., et al., Pluripotent-stem-cell-derived hepatic cells: hepatocytes and organoids for liver therapy and regeneration. Cells, 2020; $9(2)$.

7. Fox IJ, Kaufman SS, Goertzen TC, Chowdhury NR, Warkentin PI. Treatment of the Crigler-Najjar syndrome type I with hepatocyte transplantation. N Engl J Med. 1998;338:1422-6.
8. Unzu C, et al. Pharmacological induction of a progenitor state for the efficient expansion of primary human hepatocytes. Hepatology. 2019:69(5):2214-31.

9. Najimi MK, Lysy PA, Jazouli N, Abarca J, Sempoux C, Sokal EM. Adultderived human liver mesenchymal-like cells as a potential progenitor reservoir of hepatocytes. Cell Transplant. 2007;16:717-28.

10. Wang MZX, Xiong XI, Yang Z, Li P, Wang J, Sun YU, Yang Z, Hoffman RM. Bone marrow mesenchymal stem cells reverse liver damage in a carbon tetrachloride-induced mouse model of chronic liver injury. Vivo Athens Greece. 2016;30:187-93.

11. Lee SY, Kim HJ, Choi D. Cell sources, liver support systems and liver tissue engineering: alternatives to liver transplantation. Int J Stem Cells. 2015;8(1):36-47.

12. Touboul T, et al. Generation of functional hepatocytes from human embryonic stem cells under chemically defined conditions that recapitulate liver development. Hepatology. 2010;51(5):1754-65.

13. Si-Tayeb K, et al. Highly efficient generation of human hepatocyte-like cells from induced pluripotent stem cells. Hepatology. 2010;51(1):297-305

14. Yovchev Ml, et al. Repopulation of the fibrotic/cirrhotic rat liver by transplanted hepatic stem/progenitor cells and mature hepatocytes. Hepatology. 2014;59(1):284-95.

15. Zhang K, et al. In vitro expansion of primary human hepatocytes with efficient liver repopulation capacity. Cell Stem Cell. 2018;23(6):806-819 e4.

16. Qiao $S$ et al. Functional proliferating human hepatocytes: in vitro hepatocyte model for drug metabolism, excretion and toxicity. Drug Metab Dispos, 2021.

17. Suhito IR, et al. In situ label-free monitoring of human adipose-derived mesenchymal stem cell differentiation into multiple lineages. Biomaterials. 2018;154:223-33.

18. Notingher I. Raman spectroscopy cell-based biosensors. Sensors 2007;7:1343-58.

19. Butler HJ, et al. Using Raman spectroscopy to characterize biological materials. Nat Protoc. 2016;11(4):664-87.

20. Eberhardt K, et al. Advantages and limitations of Raman spectroscopy for molecular diagnostics: an update. Expert Rev Mol Diagn. 2015;15(6):773-87.

21. Notingher I, Hench LL. Raman microspectroscopy: a noninvasive tool for studies of individual living cells in vitro. Expert Rev Med Devices. 2006:3(2):215-34

22. Swain RJ. Raman microspectroscopy for non-invasivebiochemical analysis of single cells. Biochem Soc Trans. 2007;35:544-9.

23. Szafraniec E, et al. Raman spectroscopy-based insight into lipid droplets presence and contents in liver sinusoidal endothelial cells and hepatocytes. J Biophotonics. 2019;12(4):e201800290.

24. Li M, et al. Single cell Raman spectroscopy for cell sorting and imaging. Curr Opin Biotechnol. 2012;23(1):56-63.

25. Short KW, et al. Raman spectroscopy detects biochemical changes due to proliferation in mammalian cell cultures. Biophys J. 2005;88(6):4274-88.

26. Gargotti M, et al. Raman spectroscopy detects biochemical changes due to different cell culture environments in live cells in vitro. Anal Bioanal Chem. 2018;410(28):7537-50.

27. Pansare $K$, et al. Raman spectroscopy: an exploratory study to identify post-radiation cell survival. Appl Spectrosc. 2020;74(5):553-62.

28. Czamara K, et al. Impact of cell cycle dynamics on pathology recognition: Raman imaging study. J Biophotonics. 2019;12(2):e201800152.

29. Alattar $\mathrm{N}$, et al. Surface-enhanced Raman scattering for rapid hematopoietic stem cell differentiation analysis. Appl Opt. 2018;57(22):E184-9.

30. Zhang P, et al. Raman-activated cell sorting based on dielectrophoretic single-cell trap and release. Anal Chem. 2015;87(4):2282-9.

31. Choi JS, et al. Tracing hematopoietic progenitor cell neutrophilic differentiation via Raman spectroscopy. Bioconjug Chem. 2018;29(9):3121-8.

32. Wu ZT, et al. Timosaponin A3 induces hepatotoxicity in rats through inducing oxidative stress and down-regulating bile acid transporters. Acta Pharmacol Sin. 2014;35(9):1188-98.

33. Hsu CC, et al. A single-cell Raman-based platform to identify developmental stages of human pluripotent stem cell-derived neurons. Proc Natl Acad Sci USA. 2020;117(31):18412-23.

34. Jones RR, et al. Raman techniques: fundamentals and frontiers. Nanoscale Res Lett. 2019:14(1):231. 
35. Ember KJI, et al. Raman spectroscopy and regenerative medicine: a review. NPJ Regen Med. 2017;2:12.

36. Rangan S, et al. Applications of Raman spectroscopy in the development of cell therapies: state of the art and future perspectives. Analyst. 2020;145(6):2070-105.

37. Manago S, et al. Raman detection and identification of normal and leukemic hematopoietic cells. J Biophotonics. 2018;11(5):e201700265.

38. Tulsyan A, et al. A machine-learning approach to calibrate generic Raman models for real-time monitoring of cell culture processes. Biotechnol Bioeng. 2019;116(10):2575-86.

39. Li W, Li L, Hui L. Cell plasticity in liver regeneration. Trends Cell Biol. 2020;30(4):329-38.

40. Naik PP, Birbrair A, Bhutia SK. Mitophagy-driven metabolic switch reprograms stem cell fate. Cell Mol Life Sci. 2018;76(1):27-43.

41. Wanet A, et al. Connecting mitochondria, metabolism, and stem cell fate. Stem Cells Dev. 2015;24(17):1957-71.

42. Xiang G, et al. BNIP3L-dependent mitophagy accounts for mitochondrial clearance during 3 factors-induced somatic cell reprogramming. Autophagy. 2017;13(9):1543-55.

43. Fu GB, et al. Expansion and differentiation of human hepatocyte-derived liver progenitor-like cells and their use for the study of hepatotropic pathogens. Cell Res. 2019;29(1):8-22.
44. Gluchowski NL, et al. Hepatocyte deletion of triglyceride-synthesis enzyme acyl CoA: diacylglycerol acyltransferase 2 reduces steatosis without increasing inflammation or fibrosis in mice. Hepatology. 2019;70(6):1972-85.

45. Engin, A., Non-alcoholic fatty liver disease. Obes Lipotoxicity, 2017:443-467.

46. Tarlow BD, et al. Bipotential adult liver progenitors are derived from chronically injured mature hepatocytes. Cell Stem Cell. 2014;15(5):605-18.

47. Lotto J, et al. Single-cell transcriptomics reveals early emergence of liver parenchymal and non-parenchymal cell lineages. Cell. 2020;183(3):702716 e14.

48. Zhang $\mathrm{H}$, et al. A novel machine learning based approach for iPS progenitor cell identification. PLoS Comput Biol. 2019;15(12):e1007351.

\section{Publisher's Note}

Springer Nature remains neutral with regard to jurisdictional claims in published maps and institutional affiliations.
Ready to submit your research? Choose BMC and benefit from:

- fast, convenient online submission

- thorough peer review by experienced researchers in your field

- rapid publication on acceptance

- support for research data, including large and complex data types

- gold Open Access which fosters wider collaboration and increased citations

- maximum visibility for your research: over $100 \mathrm{M}$ website views per year

At BMC, research is always in progress.

Learn more biomedcentral.com/submissions 